\title{
ORIGINAL ARTICLE CBFB-MYH11/RUNX1 together with a compendium of hematopoietic regulators, chromatin modifiers and basal transcription factors occupies self-renewal genes in inv(16) acute myeloid leukemia
}

\author{
A Mandoli ${ }^{1}$, AA Singh ${ }^{1}$, PWTC Jansen ${ }^{2}$, ATJ Wierenga ${ }^{3,4}$, H Riahi $^{1}$, G Franci ${ }^{5}$, K Prange $^{1}$, S Saeed ${ }^{1}$, E Vellenga $^{3}$, M Vermeulen $^{2}$, \\ HG Stunnenberg ${ }^{1}$ and JHA Martens ${ }^{1}$
}

\begin{abstract}
Different mechanisms for CBF $\beta-M Y H 11$ function in acute myeloid leukemia with inv(16) have been proposed such as tethering of RUNX1 outside the nucleus, interference with transcription factor complex assembly and recruitment of histone deacetylases, all resulting in transcriptional repression of RUNX1 target genes. Here, through genome-wide CBF $\beta-\mathrm{MYH} 11$-binding site analysis and quantitative interaction proteomics, we found that CBF $\beta-M Y H 11$ localizes to RUNX1 occupied promoters, where it interacts with TAL1, FLI1 and TBP-associated factors (TAFs) in the context of the hematopoietic transcription factors ERG, GATA2 and PU.1/SPI1 and the coregulators EP300 and HDAC1. Transcriptional analysis revealed that upon fusion protein knockdown, a small subset of the $\mathrm{CBF} \beta-\mathrm{MYH} 11$ target genes show increased expression, confirming a role in transcriptional repression. However, the majority of $\mathrm{CBF} \beta-\mathrm{MYH} 11$ target genes, including genes implicated in hematopoietic stem cell self-renewal such as ID1, LMO1 and JAG1, are actively transcribed and repressed upon fusion protein knockdown. Together these results suggest an essential role for $\mathrm{CBF} \beta-\mathrm{MYH} 11$ in regulating the expression of genes involved in maintaining a stem cell phenotype.
\end{abstract}

Leukemia (2014) 28, 770-778; doi:10.1038/leu.2013.257

Keywords: CBF $\beta-M Y H 11$; RUNX1; histone acetylation; acute myeloid leukemia; inv(16)

\section{INTRODUCTION}

Core-binding transcription factors (CBFs) have roles in stem cell self-renewal, tissue differentiation and cancer. ${ }^{1}$ They are heterodimeric complexes consisting of two subunits, alpha and beta, in which the alpha subunit binds to DNA and is encoded by one of the RUNX1 (AML1, CBF $\alpha 2), R U N X 2$ (CBF 1$)$ and RUNX3 $(C B F \alpha 3)$ genes, whereas the beta subunit $(C B F \beta)$ is thought not to bind to DNA but to stabilize the DNA binding of the alpha subunit. RUNX1 is the CBF alpha subunit that is predominantly expressed during hematopoietic development, and both RUNX1 and CBF $\beta$ are frequently involved in chromosomal alterations associated with hematopoietic diseases, for example, in $t(8 ; 21)$ and inv(16) acute myeloid leukemia (AMLs). ${ }^{2}$

The translocation involving chromosomes 8 and 21 fuses the RUNX1 and the ETO genes, leading to the expression of AML1-ETO. Expression of the AML1-ETO oncofusion protein in hematopoietic cells results in a stage-specific arrest of maturation and increased cell survival, predisposing cells to develop leukemia. ${ }^{3}$ Whereas the AML1-ETO translocation is usually observed in AML subtype $M 2$, the inversion of chromosome 16, inv(16)(p13q22), is associated with AML-M4Eo. ${ }^{4}$ This inversion generates a chimeric gene $C B F \beta-M Y H 11$, which encodes a fusion protein between $\mathrm{CBF} \beta$ and smooth muscle myosin heavy chain (SMMHC/MYH11).
Heterozygous Cbf $\beta$-Myh11 knock-in mice are embryonic lethal, with definitive hematopoiesis blocked at the stem-cell level. Moreover, adult hematopoietic stem cells fail to differentiate to myeloid and lymphoid lineages, ${ }^{9}$ a phenotype similar to that of Run $\times 1^{-1-}$ and $C b f \beta^{-1-}$ mice ${ }^{10,11}$ and suggesting that $\mathrm{CBF} \beta-$ MYH11 is a dominant repressor of RUNX1/CBF $\beta$ function. Still, mutagenesis studies using $C b f \beta^{+/ M Y H 11}$ knock-in mice indicate that $\mathrm{CBF} \beta-\mathrm{MYH} 11$ is necessary, but not sufficient, for leukemogenesis ${ }^{12,13}$ and that additional genetic events, for example, c-KIT, RAS pathway or NDE1 mutations ${ }^{14-17}$ are required for the onset of the disease.

At the molecular level, CBF $\beta-\mathrm{MYH} 11$ has been suggested to exert its transcriptional regulator functions by several mechanisms. These include altering the normal RUNX1 transcription program through the tethering of RUNX1 outside the nucleus, interfering with transcription factor assembly, recruitment of histone deacetylases and inhibiting the RUNX1 activity., 18-20 However, many of these studies were based on in vitro and overexpression experiments. In addition, in vivo studies were hampered by the lack of knowledge on high-confidence binding sites of $\mathrm{CBF} \beta-\mathrm{MYH11}$ and identification of the target gene program.

Here we performed genome-wide-binding analysis in cell lines and patient blasts and identified many previously unknown

${ }^{1}$ Department of Molecular Biology, Faculty of Science, Nijmegen Centre for Molecular Life Sciences, Radboud University, Nijmegen, The Netherlands; ${ }^{2}$ Department of Molecular Cancer Research, UMC Utrecht, Utrecht, The Netherlands; ${ }^{3}$ Department of Hematology, University of Groningen and University Medical Center Groningen, Groningen, The Netherlands; ${ }^{4}$ Department of Laboratory Medicine University of Groningen and University Medical Center Groningen, Groningen, The Netherlands and ${ }^{5}$ Dipartimento di Biochimica, Biofisica e Patologia Generale, Seconda Università degli Studi di Napoli, Napoli, Italy. Correspondence: Dr JHA Martens, Department of Molecular Biology, Faculty of Science, Nijmegen Centre for Molecular Life Sciences, Radboud University, PO Box 9101, Nijmegen, HB 6500, The Netherlands.

E-mail: j.martens@ncmls.ru.nl

Received 27 June 2013; revised 19 August 2013; accepted 22 August 2013; accepted article preview online 4 September 2013 ; advance online publication, 27 September 2013 


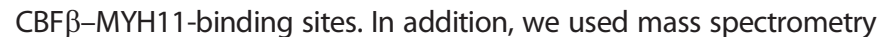
to identify new interactors of the CBF $\beta-M Y H 11 / R U N X 1$ complex. We show that CBF $\beta-M Y H 11$ binds DNA in a RUNX1-dependent manner and preferentially localizes to promoter regions. In addition, our study reveals that $\mathrm{CBF} \beta-\mathrm{MYH} 11$ operates in the context of a hematopoietic regulator complex consisting of various transcription factors linked to transcriptional activation. Finally, our study reveals that, in contrast to previous suggestions of $\mathrm{CBF} \beta-\mathrm{MYH} 11$ functioning as a transcriptional repressor, $\mathrm{CBF} \beta-\mathrm{MYH} 11$ binding can also be linked to transcriptional activation.

\section{MATERIALS AND METHODS}

\section{Chromatin immunoprecipitation (ChIP)}

Patient cells and cell lines (see Supplementary Information) were crosslinked with $1 \%$ formaldehyde for $20 \mathrm{~min}$ at room temperature, quenched with $0.125 \mathrm{M}$ glycine and washed. Sonicated chromatin (Bioruptor, Diagenode, Liege, Belgium) was centrifuged at maximum speed for $10 \mathrm{~min}$ and then incubated with specific antibodies. Beads were washed sequentially with four different wash buffers and chromatin was eluted from the beads. Protein-DNA crosslinks were reversed, after which DNA was isolated and used for quantitative PCR or sequencing analysis.

\section{Illumina high-throughput sequencing}

End repair was performed using the precipitated DNA of $\sim 6$ million cells (3-4 pooled biological replicas) using Klenow and T4 polynucleotide kinase (T4 PNK). A 3' protruding A base was generated using Taq polymerase and adapters were ligated. The DNA was loaded on gel and a band corresponding to $\sim 300 \mathrm{bp}$ (ChIP fragment + adapters) was collected. The DNA was isolated, amplified by PCR and used for cluster generation on the Illumina GAll or HiSeq genome analyzer. The 35-45 bp tags were mapped to the reference human genome using the Burrows-Wheeler Alignment Tool (BWA) or eland program allowing one mismatch. For each base pair in the genome, the number of overlapping sequence reads was determined, averaged over a $10 \mathrm{bp}$ window and visualized in the UCSC genome browser (http://genome.ucsc.edu). All ChIP-seq and RNA-seq data can be downloaded from Gene Expression Omnibus accession number GSE46044, and the bioinformatic analysis of the data is described in the Supplementary Information.

SILAC labeling, pulldown and LC-MS/MS (liquid chromatographymass spectrometry) analysis

ME-1 cells were SILAC (Stable Isotopes Labeling by Amino Acids in Cell Culture) labeled using RPMI1640 (-Arg, -Lys) medium (Gibco/lnvitrogen, Paisley, UK) supplemented with either ${ }^{13} C_{6}^{15} \mathrm{~N}_{4}$ L-arginine and ${ }^{13} \mathrm{C}_{6}^{15} \mathrm{~N}_{2}$ L-lysine (Isotec, Sigma, St Louis, MO, USA) or non-labeled L-arginine and L-lysine. Cells were cultured in SILAC medium for at least eight doublings to ensure full incorporation of the labeled amino acids. Nuclear extract preparation and pulldown were performed as previously described. ${ }^{21}$

\section{RESULTS}

CBF $\beta-M Y H 11$ binding to DNA is RUNX1 dependent

$\mathrm{CBF} \beta-\mathrm{MYH} 11$ is the oncofusion protein that results from an inversion on chromosome 16. The oncogenic activities of this protein are thought to be related to its binding to RUNX1 (also named $C B F \alpha 2$ or $A M L 1)$, thereby altering its function. Indeed, coimmunoprecipitation experiments using ME-1 cells, ${ }^{22}$ which express the CBF $\beta-M Y H 11$ variant type $A$, show that RUNX1 interacts with CBF $\beta-\mathrm{MYH} 11$ (Figure $1 \mathrm{a}$ ). CBF $\beta-\mathrm{MYH} 11$ has also been suggested to be localized both inside as well as outside the nucleus thereby diverting RUNX1 out of the nucleus and altering the normal RUNX1 transcription program. ${ }^{4}$ To examine the cellular localization of $\mathrm{CBF} \beta-\mathrm{MYH} 11$, we fractionated ME-1 cells into a nuclear and a cytoplasmic fraction. ${ }^{23}$ Western analysis of the two fractions (Figure $1 \mathrm{~b}$ ) revealed that $\mathrm{CBF} \beta-\mathrm{MYH} 11$ and $\mathrm{CBF} \beta$ are localized both in the cytoplasm as well as in the nucleus, whereas RUNX1 is primarily localized in the nucleus. These results suggest that $\mathrm{CBF} \beta-\mathrm{MYH} 11$ might indeed be involved in tethering some
RUNX1 protein outside the nucleus, but that the vast majority of RUNX1 is still nuclear localized.

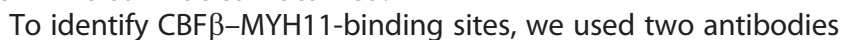
against $\mathrm{CBF} \beta$ and two antibodies against MYH11 in ChIP-seq experiments (Figure 1C; Supplementary Figures S1A, B). The use of two antibodies for each of the fusion partners that each recognize a different epitope minimizes the risk of identifying nonspecificbinding sites that arise due to cross reactivity of the antibodies. Moreover, as wt MYH11 is not expressed in ME-1 cells, ${ }^{24}$ the MYH11 antibodies allow specific identification of fusion protein binding. For all four tracks, we used model-based analysis of ChIP-Seq (MACS) with a $P$-value of $10^{-6}$ to identify signal enrichment. Overlapping the two antibodies for each of the fusion partners identified $11517 \mathrm{CBF} \beta$ - and $2145 \mathrm{MYH} 11$-binding sites (Figure 1d). Intersection of these two sets of binding regions allowed us to identify a set of 1874 regions (Supplementary Table S1) that are occupied with high confidence by the CBF $\beta-M Y H 11$ oncofusion protein. A subset of these binding sites was validated through re-ChIP experiments on targeted loci, confirming that the two parts of the fusion protein occupy the same genomic region (Figure 1e). Moreover, ChIP-seq experiments using a specific antibody against RUNX1 revealed RUNX1 colocalization with CBF $\beta-M Y H 11$ essentially at all high-confidence $\mathrm{CBF} \beta-\mathrm{MYH} 11$-binding regions (Figure 1c and Supplementary Figure S1C), reinforcing the suggestion that inv(16) leukemogenesis is RUNX1 dependent.

To validate the CBF $\beta-M Y H 11$-binding regions, we used ChIPseq to analyze the $\mathrm{CD} 34^{+}$population of a newly diagnosed inv(16) patient. Because of the low amount of patient cells, we could only perform one ChIP-seq experiment with a CBF $\beta$ and one with a MYH11 antibody (Figure 1c; Supplementary Figure S1D). Nevertheless, this analysis showed increased tag density for both $\mathrm{CBF} \beta$ as well as MYH11 at all of the high-confidence CBF $\beta-M Y H 11-$ binding sites (Figure 1f), thereby confirming the binding results obtained in the cell line. Comparing the genomic location of CBF $\beta-M Y H 11$ with PML-RAR $\alpha$ and AML1-ETO, two other AML-associated oncofusion proteins, ${ }^{25,26}$ revealed a preferential localization of $\mathrm{CBF} \beta-\mathrm{MYH} 11$ to promoter regions as compared with AML1-ETO and PML-RAR $\alpha$, which both target mostly non-promoter regions (Figure 1g). To further evaluate this finding, we partitioned our newly discovered binding sites in three categories. One category containing the $1874 \mathrm{CBF} \beta-\mathrm{MYH} 11$ (and RUNX1) binding regions, one containing all the CBF $\beta$ and RUNX1 occupied regions (CBFnoMYH) and one containing the RUNX1 only binding regions (RUNX1noCBF). Examining the genomic localization of these three categories revealed that $\sim 75 \%$ of CBF $\beta-M Y H 11 / R U N X 1$ and $C B F \beta / R U N X 1$ are enriched at transcription start sites, whereas $80 \%$ of RUNX1 only binding regions were non-promoter sites (Figure $1 \mathrm{~h}$ ), suggesting that $\mathrm{CBF} \beta-\mathrm{MYH} 11$ and $\mathrm{CBF} \beta$ are mainly involved in regulating promoter activity, whereas RUNX1 can also regulate the activities of enhancer regions. Moreover, the results suggest that the interaction with RUNX1 alone cannot explain the localization of $\mathrm{CBF} \beta$ and $\mathrm{CBF} \beta-\mathrm{MYH} 11$, as this would result in a different, more RUNX1-like genomic distribution. Hence additional factors are in place to guide $\mathrm{CBF} \beta$ and $\mathrm{CBF} \beta-\mathrm{MYH} 11$ to promoter regions.

\section{TAFs bind the CBF $\beta-M Y H 11 /$ RUNX1 complex}

Next we set out to assess which proteins act together with CBF $\beta$ $\mathrm{MYH} 11$ in transcriptional regulation. As CBF $\beta-\mathrm{MYH} 11$ in vivo is localized to RUNX1-binding sites, it allowed us to identify the CBF $\beta-M Y H 11$ protein complex using DNA pull-down analysis followed by mass spectrometry. For this we performed DNA pulldown experiments using a specific nucleotide sequence bound by $\mathrm{CBF} \beta-\mathrm{MYH} 11$ in ME- 1 cells (Supplementary Figure S2A) that contains the RUNX1 core consensus motif TGTGGT (RUNX1 oligo) and a control sequence with a scrambled RUNX1 motif (control oligo) (Figure 2a). We could show that the oligonucleotide with 
a

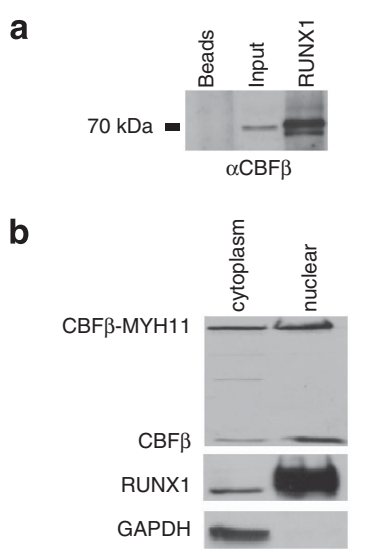

C

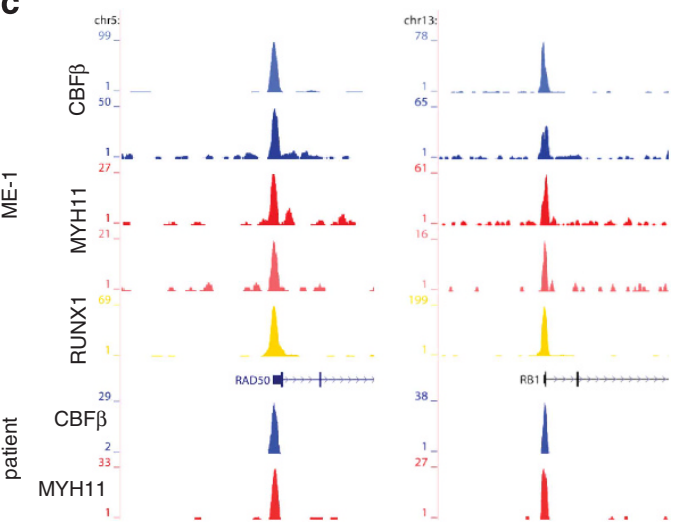
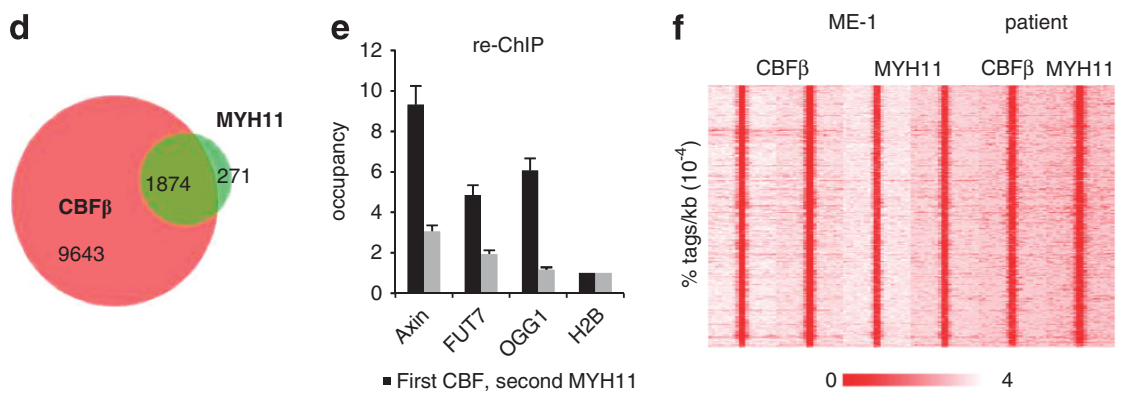

- First CBF, second no ab
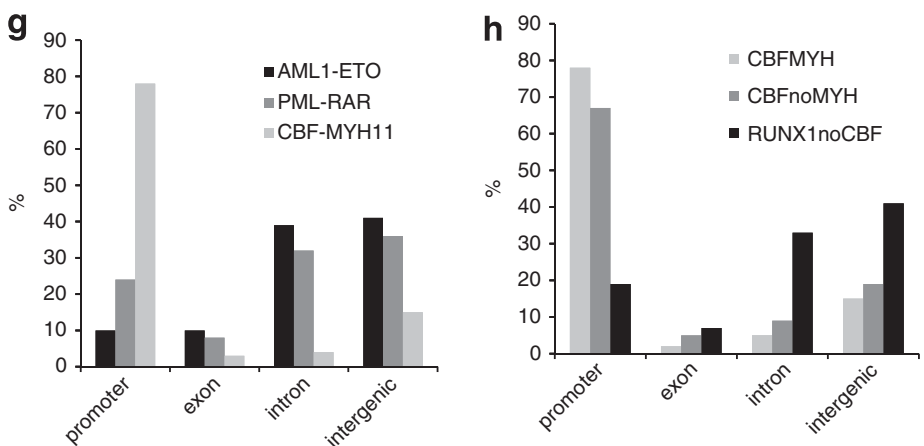

Figure 1. Genome-wide binding of CBF $\beta-M Y H 11$. (a) Coimmunoprecipitation of CBF $\beta-M Y H 11$ with RUNX1. Immunoprecipitations were performed in ME-1 cells using immunoglobulin G (beads) and RUNX1 antibodies and analyzed by western using CBF $\beta$ antibodies. (b) Western analysis of the cytoplasmic and nuclear fraction of ME-1 cells using antibodies recognizing CBF $\beta$ and RUNX1 and a control GAPDH antibody to validate the fractionation. (c) Overview of the RAD50 and RB1 CBF $\beta-M Y H 11$-binding sites in ME-1 cells and a patient blast. In blue, the CBF $\beta$ ChIP-seq data are plotted, in red the MYH11 and in yellow the RUNX1 data. (d) Venn diagram representing the overlap of CBF $\beta$ and MYH111binding sites in ME-1 cells. (e) Re-ChIP experiment validating CBF $\beta$ and MYH11 binding to the same locus. Three binding sites were selected and validated for $\mathrm{CBF} \beta / \mathrm{MYH} 11$ binding by re-chip using CBF $\beta$ antibodies in the first round of ChIP followed by a second round using MYH11 and no antibodies (f) Heat map displaying CBF $\beta$ and MYH11 tag densities in ME-1 an inv(16) patient cells at the high-confidence CBF $\beta$ MYH11-binding sites identified in ME-1 cells. (g) Distribution of the CBF $\beta$-MYH11, AML1-ETO and PML-RAR $\alpha$-binding site locations relative to RefSeq genes. Locations of binding sites are divided in promoter ( $-500 \mathrm{bp}$ to the transcription start site), exon, intron and intergenic (everything else). (h) Distribution of CBF $\beta-M Y H 11 / R U N X 1, C B F \beta / R U N X 1$ and RUNX1-binding site locations relative to RefSeq genes. Location are as in (g).

the RUNX1 motif efficiently pulls down CBF $\beta-M Y H 11$ as well as CBF $\beta$ from a ME-1 cell lysate, whereas the control sequence showed significantly reduced affinity for $\mathrm{CBF} \beta-\mathrm{MYH11}$ (Figure $2 \mathrm{~b}$ ), confirming in vitro that $\mathrm{CBF} \beta-\mathrm{MYH} 11$ binding to DNA is largely RUNX1 dependent.

To characterize the interactome of the CBF $\beta-M Y H 11 / R U N X 1$ complex, we used previously described SILAC-based technology, ${ }^{21}$ using extracts derived from ME-1 cells grown in 'light' or 'heavy' medium, incubated with oligonucleotides containing the RUNX1 or a scrambled RUNX1 motif (see Materials and Methods). Of the $>900$ identified proteins, over 40 had highly significant ratios
( $>4$ ) (Figure 2c; Supplementary Table S1) indicating specific binding to the CBF $\beta-M Y H 11 / R U N X 1$ complex, whereas over 100 additional proteins, including previously identified RUNX1 interactors TAL1, FLI1 and BMI1, ${ }^{27}$ had lower ratio's $(>2)$ and might represent transient- or context-dependent interactions (Supplementary Table S1).

Interestingly, our pull-down analysis also suggested interaction of CBF $\beta-M Y H 11$ with eight different TAFs (TBP-associated factors), TAF1, 3, 4, 5, 6, 7, 9 and 10 (Figure 2c; Supplementary Table S1). Subsequent coimmunoprecipitation experiments using ME-1 cells showed an interaction between CBF $\beta-$ MYH11 and TAF7 
a
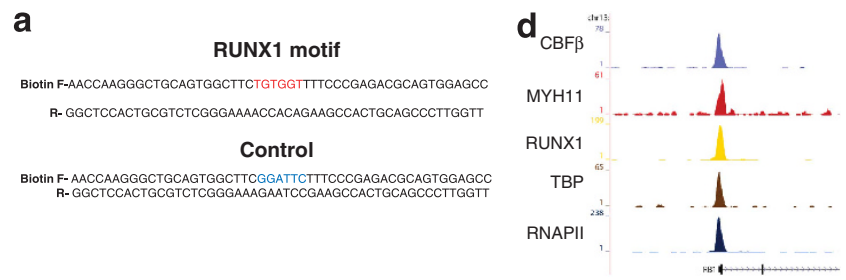

b

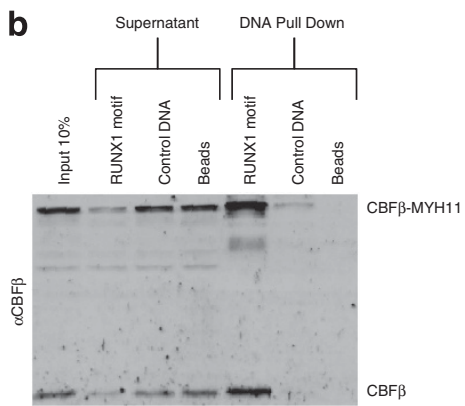

e

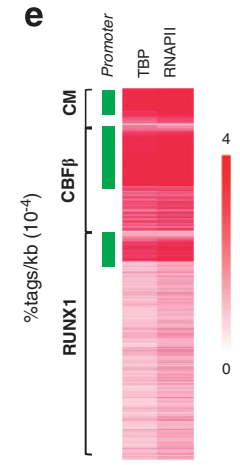

C

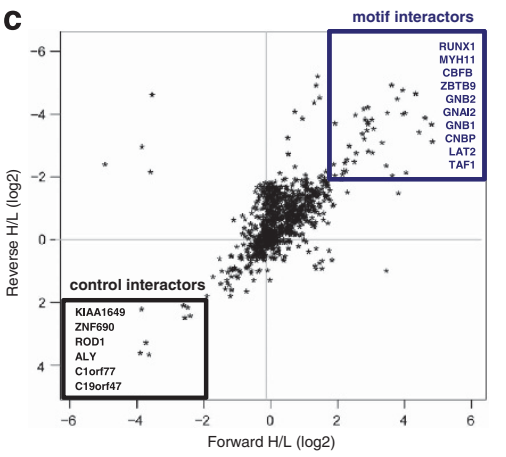

f

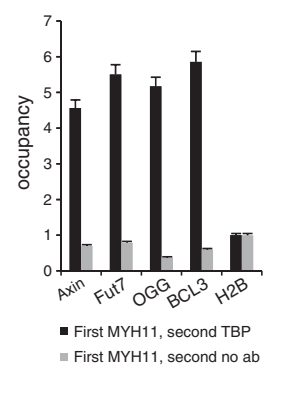

Figure 2. Identification of CBF complex components. (a) DNA sequence of the oligo's used in the pull-down analysis. (b) Western analysis of a DNA pulldown in ME-1 cells using a CBF $\beta$ antibody. $\mathrm{CBF} \beta$ and $\mathrm{CBF} \beta-\mathrm{MYH} 11$ are enriched in the pulldown with the RUNX1 motif in comparison with the control. (c) Dotplot showing the result of the pull-down mass spectrometry experiment. Proteins are plotted by their SILAC-ratios in the forward ( $x$ axis) and reverse (y axis) SILAC experiment. Specific interactors of the RUNX1 pulldown lie in the upper right quadrant. (d) Overview of the RB1

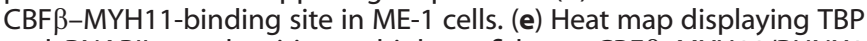
and RNAPII tag densities at high-confidence CBF $\beta-M Y H 11 / R U N X 1$ $(C M), C B F \beta / R U N X 1(C B F \beta)$ and RUNX1 only binding sites. Highest occupancy is observed at promoter regions bound by CBF $\beta-\mathrm{MYH} 11$. (f) Re-ChIP experiment validating CBF $\beta-\mathrm{MYH} 11 / \mathrm{TBP}$ binding to the same locus. Three binding sites were selected and validated for CBF $\beta-M Y H 11 /$ TBP binding by re-ChIP using MYH11 antibodies in the first round of ChIP followed by a second round using TBP and no antibodies.

(Supplementary Figure S2B), corroborating the pull-down results. Together these results suggest that interaction with TAFs might guide the localization of $\mathrm{CBF} \beta$ or the fused $\mathrm{CBF} \beta-\mathrm{MYH} 11$ to promoter sites.

As TAFs form a stable complex with TBP and RNAPII to form a preinitiation complex, we wondered whether in vivo these two factors would bind similar genomic regions as the CBF $\beta-M Y H 11$ complex. Indeed, genome-wide analysis revealed the occupancy of CBF $\beta-M Y H 11 / R U N X 1, T B P$ and RNAPII at similar genomic regions (Figure $2 \mathrm{~d}$ ). Moreover, further analysis of TBP and RNAPII occupancy at CBF $\beta-\mathrm{MYH} 11$ (CM) regions as compared with RUNX1/CBF $\beta$ (CBF $\beta)$ and RUNX1 only regions revealed highest

levels of TBP and RNAPII at CBF $\beta-$ MYH1 1 sites located in promoter regions (Figure $2 \mathrm{e}$ ).

To further validate that they bind the same genomic regions, we performed re-ChIP experiments. This confirmed TBP and CBF $\beta-$ MYH11 binding to the same genomic loci (Figure 2f). Together these results suggest that the CBF $\beta-M Y H 11 / R U N X 1$ complex interacts with basal transcriptional factors and occupies similar genomic regions.

CBF $\beta-M Y H 11 /$ RUNX1 bound regions are occupied by ETS factors, TAL1, GATA2 and HEB

To examine whether CBF $\beta-M Y H 11$-binding sites harbor specific DNA elements, we investigated the presence of consensus binding motifs for various hematopoietic regulators such as E-box proteins, GATA and ETS factors, TAL1 and RUNX. This analysis revealed that most binding sites harbor DNA motifs for RUNX1, ETS and E-box factors (Figure 3a), while also TAL1, GATA and $\mathrm{NFKB}$ consensus binding sequences can be found in a high number of sites. These results suggest that $\mathrm{CBF} \beta-\mathrm{MYH} 11$ might function in the context of other hematopoietic regulators.

The pull-down analysis suggested interaction of the CBF complex with TAL1 and the ETS factor FLI1, in line with the presence of the RUNX1, ETS and TAL1 motif at CBF $\beta-M Y H 11-$ binding sites. Our motif analysis further revealed the presence of the E-box and GATA motif at CBF $\beta-M Y H 11-$ binding sites. To investigate whether TAL1, E-box and GATA factors are present at $\mathrm{CBF} \beta-\mathrm{MYH} 11$ bound regions, we extended our ChIP-seq analysis and included specific antibodies to TAL1, GATA2, the GATA factor highest expressed in ME-1 cells, HEB, a protein previously implicated in CBF leukemogenesis, ${ }^{26,28,29}$ and also included several ETS factors, such as ELF1, FLI1, ERG and PU.1/SPI1 in our analysis. This revealed the increased occupancy of all factors at the CBF $\beta-M Y H 11 / R U N X 1$ occupied promoters of the C8ORF55 and SOCS1 genes (Figure 3b) whereas, in contrast, not all factors were enriched at the CBF $\beta / R U N X 1$ occupied TAL1 or RUNX1 occupied PSMC3 gene. For all transcription factors, we identified the genome-wide binding regions using model-based analysis of ChIP-Seq (MACS), and the resulting peak files were, together with the different sets of CBF-binding sites (CBF $\beta-M Y H 11 / R U N X 1$, CBF $\beta /$ RUNX1 and RUNX1 only), used as input for unsupervised segmentation analysis using ChromHMM. ${ }^{30}$ ChromHMM is based on a multivariate hidden Markov model that allows modeling of the presence or absence of a transcription factor by integrating multiple data sets to discover de novo the major re-occurring combinatorial and spatial patterns of transcription factors. Here, it allowed the identification of 23 distinct transcription factor co-occupancy patterns within the inv(16) cells (Figure 3c). In line with our observations at the TAL1 and PSMC3 genes, various transcription factor patterns could be detected at CBF $\beta / R U N X 1$ or at RUNX1 only occupied regions (Figure $3 \mathrm{c}$ ). In contrast, at the CBF $\beta-M Y H 11 / R U N X 1$ occupied regions (state 9), enrichment for all transcription factors was found (Figure $3 \mathrm{c}$ and Supplementary Figure S3), suggesting that CBF $\beta-\mathrm{MYH} 11$ functions in the context of many other hematopoietic transcription factors.

To further validate that $\mathrm{CBF} \beta-\mathrm{MYH} 11$ binds the same genomic regions as other hematopoietic regulators, we performed re-ChIP experiments. This confirmed that ELF1, FLI1, PU.1, ERG and CBF $\beta-$ MYH11 bind the same genomic locus (Figure 3d).

\section{HATs and HDACs regulate CBF $\beta-M Y H 11$ occupied regions} CBF $\beta-M Y H 11$ has been reported to recruit HDACs to its target sites, ${ }^{31}$ similar as for other oncofusion proteins such as AML1-ETO and PML-RAR $\alpha$. To assess whether CBF $\beta-M Y H 11$ binding correlates with histone (de)acetylation, we performed ChIP-seq analysis using an antibody that recognizes $\mathrm{H} 3$ acetylation. Examining the average profile of this histone mark at our previously defined binding sites revealed highest $\mathrm{H} 3$ acetylation 

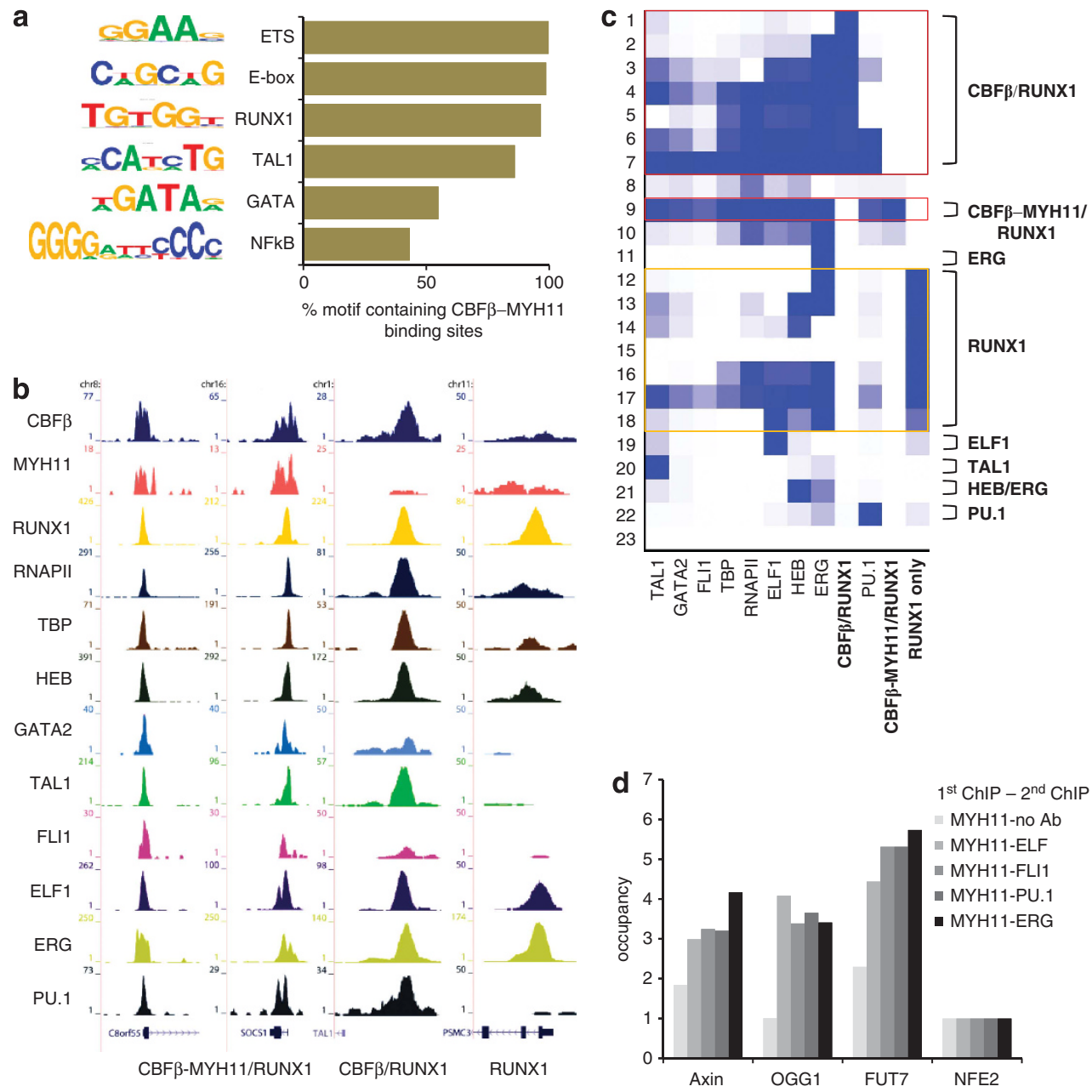

Figure 3. Transcription factor occupancy at CBF $\beta-M Y H 11$-binding sites. (a) Diagram depicting the percentage of CBF $\beta-\mathrm{MYH} 11-\mathrm{binding}$ sites harboring a hematopoietic regulator consensus binding sequences. (b) Overview of the C8ORF55 and SOCS1 CBF $\beta-M Y H 11 / R U N X 1$, the TAL 1 CBF $\beta$ /RUNX1 and the PSMC3 RUNX1-binding site in ME-1 cells. ChIP-seq data are plotted for CBF $\beta$, MYH11, RUNX1, RNAPII, TBP, HEB, GATA2, TAL1, FLI1, ELF1, ERG and PU.1. (c) ChromHMM segmentation analysis of the transcription factor binding sites in ME-1 cells. (d) Re-ChIP experiment validating CBF $\beta-M Y H 11 / T F$ binding to the same locus. Three binding sites were selected and validated for CBF $\beta-M Y H 11 / T F$ binding by re-chip using MYH11 antibodies in the first round of ChIP followed by a second round using ELF, FLI1, PU.1, ERG and no antibodies.

at the 1874 CBF $\beta-M Y H 11 / R U N X 1-$ binding sites (CBFMYH11), whereas the sets of unique CBF $\beta /$ RUNX1 (CBF) and RUNX1 only sites harbor lower levels of $\mathrm{H} 3$ acetylation (Figure 4a).

Histone acetylation levels have been suggested to be the result of the dynamic interplay of histone acetyl tranferases (HATs) and histone deacetylases (HDACs). ${ }^{32-35}$ To examine whether both enzymatic activities are recruited to $\mathrm{CBF} \beta-\mathrm{MYH} 11$ sites, we performed ChIP-seq using specific antibodies against HDAC1 and the HAT EP300. This analysis revealed the presence of both EP300 and HDAC1 at the CBF $\beta-M Y H 11$ sites at the HDAC 1 and HM13 genes (Figure 4b). Intersection of the EP300 and HDAC1 sites confirmed that on a genome-wide scale both HAT and HDAC activities are recruited together at many sites (Figure 4c). Examining the occupancy of EP300 and HDAC1 as well as H3 acetylation specifically at all CBF $\beta-\mathrm{MYH} 11$-binding sites revealed increased levels at all high-confidence sites (Figure 4d). Together these results suggest that $\mathrm{H} 3$ acetylation levels at CBF $\beta-M Y H 11$ are the result of the activities of counteracting HAT and HDAC proteins.

CBF $\beta-M Y H 11$ binds at the promoters of active genes

As our results reveal that $\mathrm{CBF} \beta-\mathrm{MYH} 11$ (and $\mathrm{CBF} \beta$ ) targets promoter regions that harbor increased $\mathrm{H} 3$ acetylation, we wondered whether CBF $\beta-\mathrm{MYH} 11$ target genes are expressed. For this we assigned genes to each binding site and analyzed the expression of these target genes through RNA-seq. RPKM (reads per kilobase exon per million tags sequenced) analysis revealed that although the expression of genes associated with CBF $\beta-M Y H 11 / R U N X 1$ occupied promoters can vary extensively (Figure $5 \mathrm{a}$, outer whiskers in boxplot), RPKM values are generally higher in this context than for genes that bind only CBF $\beta /$ RUNX1 $(\mathrm{CBF} \beta)$ or RUNX1. As RPKM measurements are derived from RNA, which could have been alternatively regulated through post transcriptional processes, we decided to also examine RNAPII occupancy as a measurement for ongoing transcription. ${ }^{36}$ Analysis of the same gene sets revealed highest RNAPII occupancy at genes targeted by CBF $\beta-$ MYH11 (Figure 5b), whereas CBF $\beta$ / RUNX1 and RUNX1 only target genes have reduced RNAPII occupancy.

Finally, we analyzed the expression status using available microarray expression data ${ }^{37}$ of the target genes in patients $(n=33)$ harboring an inv(16) translocation. This analysis confirmed that $\mathrm{CBF} \beta-\mathrm{MYH} 11$ target genes are higher expressed than $\mathrm{CBF} \beta /$ RUNX1 or RUNX1 only target genes (Figure $5 \mathrm{c}$ ). Together, these results suggest that although $\mathrm{CBF} \beta-\mathrm{MYH} 11$ has been suggested to repress transcriptional activity, $\mathrm{CBF} \beta-\mathrm{MYH} 11$ binding can also be associated with gene activity. 
a

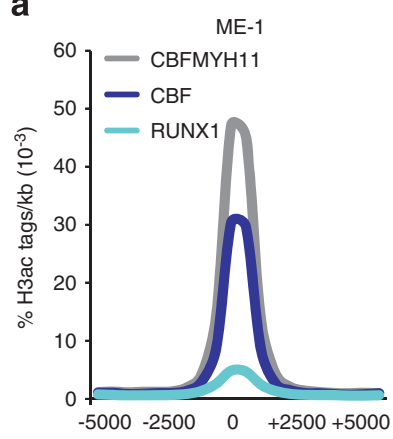

C

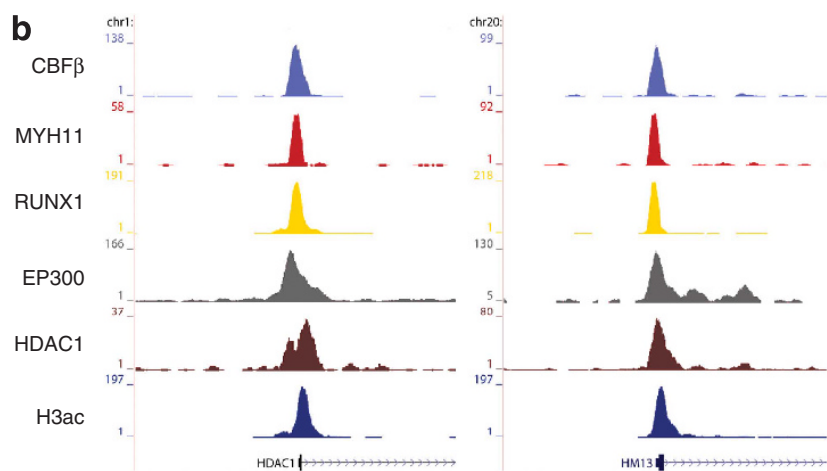

d EP300 HDAC1 H3ac
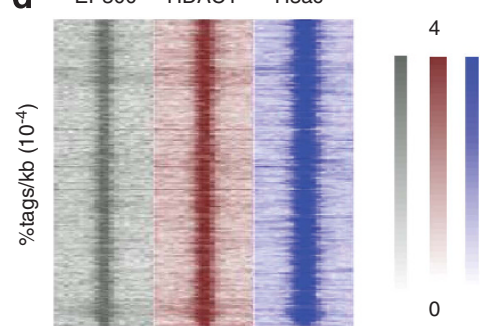

Figure 4. $\mathrm{CBF} \beta-\mathrm{MYH} 11$ occupied regions are hyperacetylated. (a) Median $\mathrm{H} 3$ acetylation profile over CBF $\beta-\mathrm{MYH} 11 / \mathrm{RUNX} 1$, CBF $\beta / \mathrm{RUNX} 1$ (CBF) and RUNX1-binding sites. (b) Overview of the HDAC1 and HM13 CBF $\beta-M Y H 11$-binding sites in ME-1 cells, showing the binding of both EP300 and HDAC1 as well as increased levels of H3 acetylation. (c) Venn diagram representing the overlap of EP300- and HDAC1-binding sites in ME-1 cells. (d) Heat map displaying EP300, HDAC1 and H3ac tag densities at high-confidence CBF $\beta$-MYH11-binding sites.

a

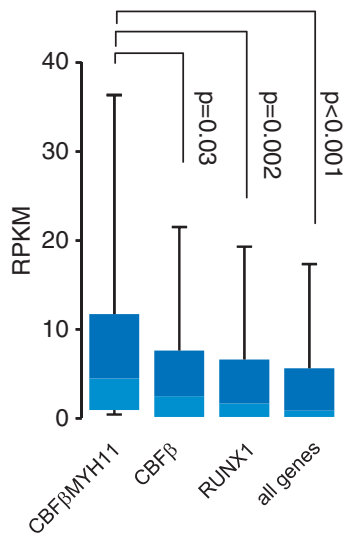

ME-1 b

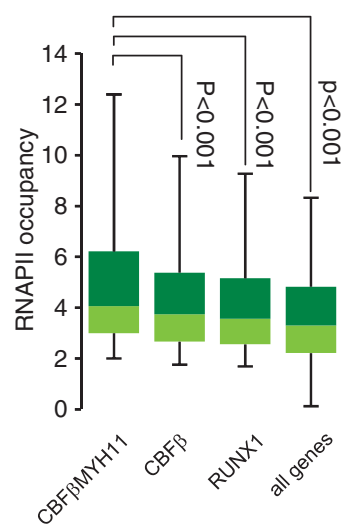

ME-1
C
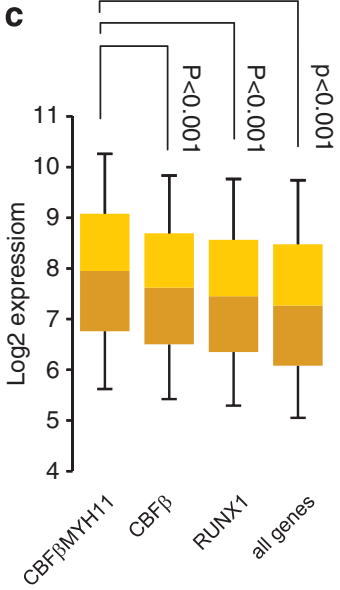

$\operatorname{lnv}(16)$ patient $(n=33)$

Figure 5. $\mathrm{CBF} \beta-\mathrm{MYH} 11$ occupied regions correlate with increased gene activity. (a) Boxplot showing RPKM values of CBF $\beta-M Y H 11 / \mathrm{RUNX} 1$, $\mathrm{CBF} \beta /$ RUNX1 (CBF $\beta$ ) and RUNX1 target genes in ME-1 cells. (b) Boxplot showing RNAPII tag density at CBF $\beta-M Y H 11 / R U N X 1, \mathrm{CBF} \beta / \mathrm{RUNX} 1$ and RUNX1 target genes in ME-1 cells. (c) Boxplot showing log 2 expression values of CBF $\beta-M Y H 11 / R U N X 1$, CBF $\beta / R U N X 1$ and RUNX1 target genes in inv(16) patient blasts $(n=33)$.

$\mathrm{CBF} \beta-\mathrm{MYH} 11$ is involved in gene activation and repression

To further examine the effects of $\mathrm{CBF} \beta-\mathrm{MYH} 11$ binding on gene expression, we made use of an inducible U937 cell line that upon tetracycline (Tet) depletion expressed CBF $\beta-$ MYH11 (Tet-off). ${ }^{38}$ Indeed, $72 \mathrm{~h}$ after Tet was removed the expression of CBF $\beta-M Y H 11$ was detected both at the level of RNA (Figure 6a) and protein (Figure $6 \mathrm{~b}$ ), although lower as observed in the ME-1 cell line. ChIP-seq using antibodies against CBF $\beta$ and MYH11 after CBF $\beta-M Y H 11$ expression allowed the identification of 2312 novel binding sites occupied by both $\mathrm{CBF} \beta$ and $\mathrm{MYH} 11$ (Figure $6 \mathrm{c}$ ). Assigning these binding sites to genes and subsequent RNA-seq analysis revealed that, of the 263 genes twofold or more upregulated or downregulated, $67 \%$ are twofold or more upregulated (Figure 6d), whereas 33\% are lowly expressed, suggesting that $\mathrm{CBF} \beta-\mathrm{MYH} 11$ is involved both in transcriptional activation and repression.

To examine the effect of CBF $\beta-\mathrm{MYH} 11$ knockdown on inv(16) cells, we constructed a CBF $\beta-M Y H 11$ inducible knockdown system using ME-1 cells. Reverse transcription-quantitative PCR and western analysis of ME-1 cells carrying an inducible CBF $\beta$ MYH11 knockdown construct revealed, after induction, decreased RNA and protein levels of CBF $\beta-M Y H 11$ in the ME- 1 cells carrying 

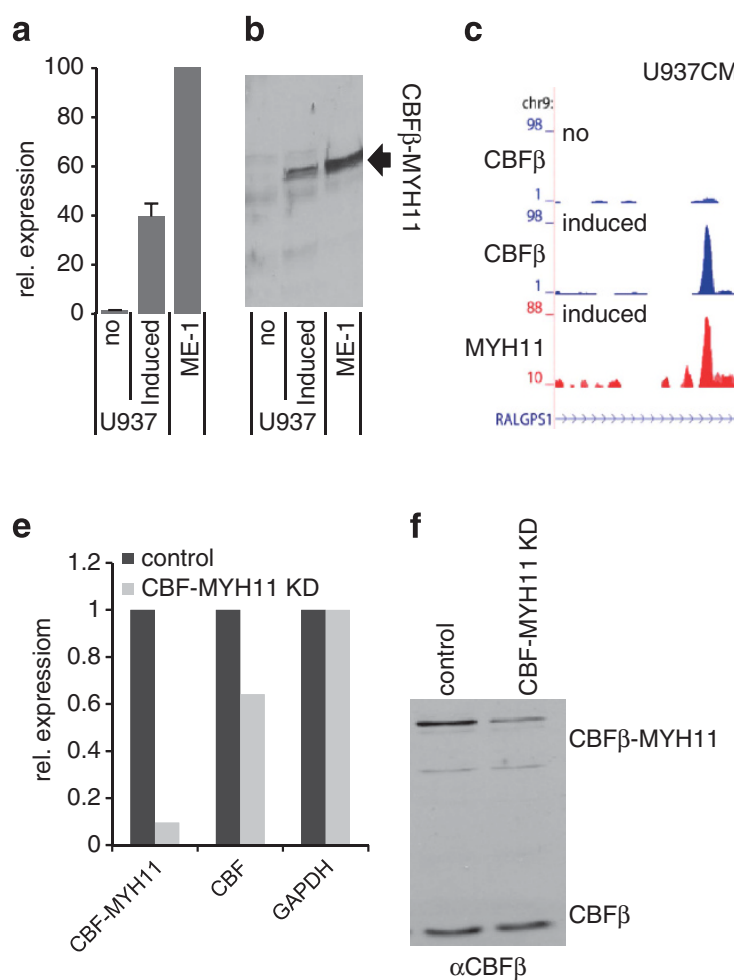

g

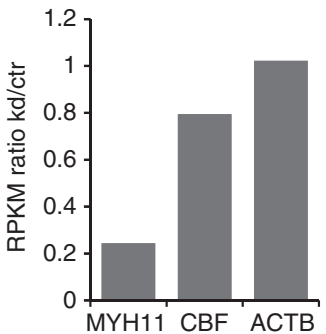

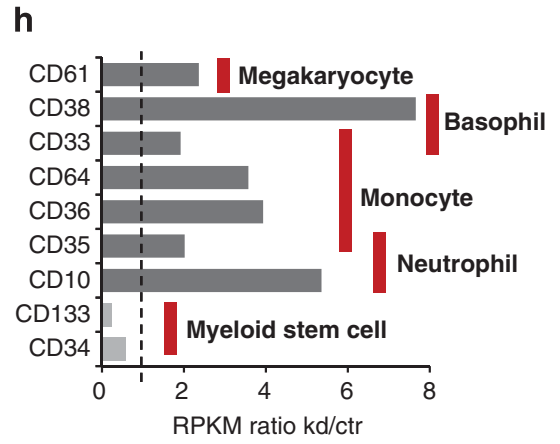

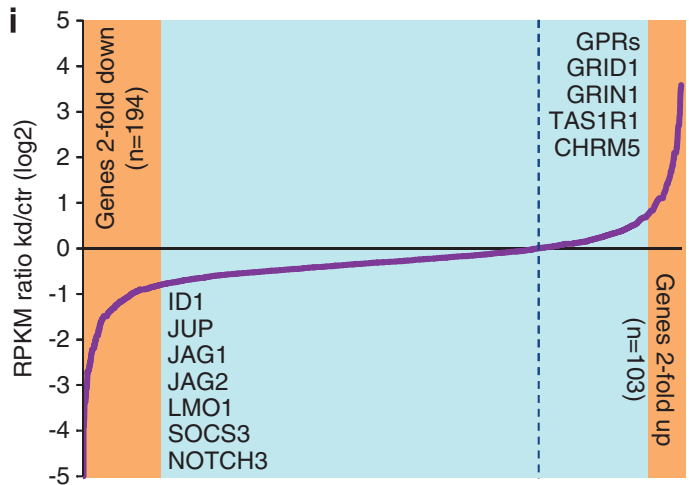

Figure 6. $\mathrm{CBF} \beta-\mathrm{MYH} 11$ is involved in gene activation and repression. (a) Reverse transcription-quantitative $\mathrm{PCR}$ (RT-qPCR) analysis of CBF $\beta-$ MYH11 expression in U937 TET inducible CBF $\beta-$ MYH11 cells (U937CM) and ME-1 cells. (b) Western blot analysis of CBF $\beta-M Y H 11$ expression in U937 TET inducible CBF $\beta$-MYH11 (U937CM) and ME-1 cells. (c) Overview of the RALGPS1 CBF $\beta-M Y H 11$-binding site in U937CM cells. In blue the CBF $\beta$ ChIP-seq data are plotted in induced and uninduced U937CM cells and in red the MYH11 ChIP-seq data in induced U937CM cells. (d) Heatmap display of the log2 ratio of CBF $\beta-\mathrm{MYH} 11$ target genes after and before the induction of oncofusion protein expression. Only results for the 263 genes twofold or more up- or downregulated are shown. (e) RT-qPCR analysis of CBF $\beta-M Y H 11$ and CBF $\beta$ expression in ME-1 cells expressing a CBF $\beta-M Y H 11$ knockdown hairpin without (control) or after induction. (f) Western analysis of CBF $\beta-\mathrm{MYH} 11$ and $\mathrm{CBF} \beta$ expression in ME-1 cells expressing a CBF $\beta-\mathrm{MYH} 11$ knockdown hairpin without and after induction. (g) RNA-seqbased RPKM ratio of CBF $\beta-M Y H 11$ (MYH11), CBF $\beta$ (CBF) and $\beta$-actin (ACTB) of CBF $\beta-M Y H 11$ knockdown ME- 1 cells versus an uninduced control. (h) RPKM ratio of various CD markers of CBF $\beta-M Y H 11$ knockdown ME-1 cells versus an uninduced control. The cell type for which the $\mathrm{CD}$ markers are indicative are mentioned on the right. (i) RPKM ratio of CBF $\beta-\mathrm{MYH} 11$ knockdown ME- 1 cells versus an uninduced control of all CBF $\beta-\mathrm{MYH} 11$ target genes. A total of 194 target genes are twofold or more downregulated and 103 target genes are more then twofold upregulated after CBF $\beta-\mathrm{MYH} 11$ knockdown.

the knockdown hairpin (Figures 6e and f). Subsequent genomewide transcriptome analysis using RNA-seq confirmed lower $C B F \beta-M Y H 11$ RPKM levels after knockdown (Figure $6 \mathrm{~g}$ ).

Upon CBF $\beta-M Y H 11$ knockdown, we observed increased attachment of cells to the culture dish suggesting the initiation of a differentiation/cell adherens program (Supplementary Figure S4A). To further assess this knockdown phenotype, we examined the expression of $C D$ markers. This revealed that ME-1 cells with lowered CBF $\beta-\mathrm{MYH} 11$ have decreased levels of myeloid stem cell marker genes such as CD34 and CD133 (Figure 6h), whereas various markers for differentiated myeloid cell types are increased (Figure 6h; Supplementary Table S1), suggesting that CBF $\beta$ MYH11 knockdown results in a more differentiated myeloid phenotype. In addition, we examined which $\mathrm{CBF} \beta-\mathrm{MYH} 11$ target genes are differential expressed upon CBF $\beta-M Y H 11$ knockdown. Using a twofold change cutoff, 194 genes were identified for which the expression is lower upon knockdown, whereas 103 CBF $\beta-M Y H 11$ target genes are higher expressed (Figure $6 i_{\text {; }}$ Supplementary Table S1), suggesting that the oncofusion protein is involved both in gene expression and repression. Apart from 
CBF $\beta-\mathrm{MYH} 11$ target genes also many $\mathrm{CBF} \beta / \mathrm{RUNX} 1$ and RUNX1 only target genes were differentially expressed (Supplementary Figure S4B; Supplementary Table S1), suggesting that the effects of $\mathrm{CBF} \beta-\mathrm{MYH} 11$ extend to the complete CBF gene network.

Although for the CBF $\beta-\mathrm{MYH} 11$ target genes that were highly expressed, many receptors, such as GPRs, CHRM5, GRIN1 and GRID1, were detected (Figure 6i), genes that were lower expressed include suppressors of cytokine signaling (SOCS3) and members of the WNT (JUP) and NOTCH (JAG1, JAG2 and NOTCH3) pathways (Supplementary Figure S4C). Interestingly, JAG1, but also LMO1 and ID1, have been implicated in hematopoietic stem cell expansion, ${ }^{39-42}$ suggesting that reduced expression of these genes in CBF $\beta-M Y H 11$ knockdown cells affects its self-renewal capacity. Indeed, hypergeometric testing of a combined set of self-renewal pathways involved in myeloid malignancies ${ }^{43}$ revealed significant enrichment $\left(P\right.$-value $\left.1.59 \mathrm{e}^{-4}\right)$ (Supplementary Figure S4C) for genes downregulated upon CBF $\beta-\mathrm{MYH} 11$ knockdown.

\section{DISCUSSION}

Many breakpoints involved in specific chromosomal translocations have been cloned over the years. In most cases, however, the role of the chimeric oncofusion proteins in tumorigenesis has not been elucidated. In the case of AML, our analysis of PML-RAR $\alpha$ and AML1ETO were among the first to report on the genome-wide actions of oncofusion proteins. ${ }^{25,26,44}$ Here, we analyzed the genome-widebinding pattern of $\mathrm{CBF} \beta-\mathrm{MYH} 11$ and its interplay with other regulators of hematopoiesis in cell lines and patient primary blasts.

As CBF $\beta-\mathrm{MYH} 11$ has been suggested to localize primarily in the cytoplasm ${ }^{45,46}$ thereby tethering RUNX1 outside the nucleus, we first examined the cellular localization of both proteins through fractionation experiments. This revealed $\mathrm{CBF} \beta-\mathrm{MYH} 11$ presence both in the cytoplasm as well as in the nucleus, whereas RUNX1 was predominantly localized in the nucleus, suggesting that $\mathrm{CBF} \beta-\mathrm{MYH} 11$ could tether some RUNX1 outside the nucleus, but that it also has a role within the nucleus.

To identify CBF $\beta-\mathrm{MYH} 11$ binding, we used two antibodies specifically recognizing the CBF $\beta$ part as well as two antibodies recognizing the $\mathrm{MYH} 11$ part of the $\mathrm{CBF} \beta-\mathrm{MYH} 11$ protein in ChIP-seq and identified 1874 high confidence, mostly promoter binding sites in ME-1 cells. In addition, we analyzed genome-wide $\mathrm{CBF} \beta-\mathrm{MYH} 11$ binding in a patient blast and could validate enrichments of both $\mathrm{CBF} \beta$ and $\mathrm{MYH11}$ at all high-confidence binding sites. Moreover, we could detect RUNX1 at all $\mathrm{CBF} \beta-\mathrm{MYH} 11$-binding sites in line with previous reports suggesting that the presence of RUNX1 is required for CBF $\beta-M Y H 11$ binding.

We used a SILAC-based mass spectrometry approach to identify components of the CBF $\beta-M Y H 11$ complex and confirmed previous observations on the presence of TAL1 and FLI1 in the CBF RUNX1/CBF $\beta$ complex. In addition, the interaction study revealed the presence of eight TAFs. As CBF $\beta-M Y H 11$ localizes primarily to promoter regions, these results suggest that the preferential localization of CBF $\beta-\mathrm{MYH} 11$ to promoters may be a result of the interaction with TAFs.

The presence of binding motifs for all major hematopoietic regulators in $\mathrm{CBF} \beta-\mathrm{MYH} 11$-binding regions prompted further investigation on the presence of hematopoietic regulators. This investigation, which included the analysis using specific HEB, GATA2, ERG, PU.1 and ELF1 antibodies in ChIP-seq, revealed the presence of all these important regulators at $\mathrm{CBF} \beta-\mathrm{MYH} 11$ occupied regions. Although it was previously suggested that CBF $\beta-M Y H 11$ interferes with the RUNX1 transcription program through steric hindrance of transcription factor complex assembly $^{4,5}$ the MYH11 moiety of CBF $\beta-M Y H 11$ apparently does not preclude the interaction and co-occupancy of the transcription factors examined in this study.

In addition, our analysis revealed that at the epigenetic level $\mathrm{CBF} \beta-\mathrm{MYH} 11$ occupied regions are enriched for $\mathrm{H} 3$ acetylation and most likely regulated by the balanced interplay of the antagonistic activities of histone-modifying enzymes such as HATs and HDACs (but most likely also methyltransferases and demethylases). ${ }^{32-35}$ Together these results suggest that genes important for maintaining leukemogenic potential (as evident from the targeting of $\mathrm{CBF} \beta-\mathrm{MYH} 11$ to these regions) are regulated by many different transcription factors and coregulators and that most likely their interplay determines the transcriptional output.

Previously, CBF $\beta-\mathrm{MYH} 11$ has been proposed to act as a transcriptional repressor of its target genes. To assess this, we examined the expression of CBF $\beta-M Y H 11$ target genes in the ME-1 inv(16) cell line, in a U937 cell line harboring an inducible CBF $\beta-M Y H 11$ construct and in patient cells. Comparing all genes targeted by CBFs (CBF $\beta-M Y H 11 / R U N X 1, C B F \beta / R U N X 1$ and RUNX1 only), we found those that have $\mathrm{CBF} \beta-\mathrm{MYH} 11$ binding to be on average the highest expressed, whereas those genes bound by CBF $\beta / R U N X 1$ or RUNX1 alone were lowly expressed, suggesting a role for $\mathrm{CBF} \beta-\mathrm{MYH} 11$ in transcriptional activation. CBF $\beta-\mathrm{MYH} 11$ overexpression as well as knockdown confirmed that subsets of target genes are repressed by the oncofusion protein, but that for a large set $\mathrm{CBF} \beta-\mathrm{MYH} 11$ is involved in maintaining increased transcriptional activity. What causes this difference is currently unclear, but it might be dependent on the presence of not yet identified proteins, interactions with enhancer elements, signaling cascades and/or spatial localization. Among the genes that are actively transcribed in inv(16) cells and repressed after CBF $\beta-M Y H 11$ knockdown are JAG1, LMO1 and ID1. Interestingly, enforced expression of $L M O 1$ induces leukemia's, ${ }^{39}$ and $L M O 1$ null mutations in mice can lead to severe reduction of steady-state hematopoietic stem cell numbers, ${ }^{41}$ a phenotype also observed in ID1-deficient hematopoietic stem cells. ${ }^{42}$ Finally, inducing JAG1 expression results in the expansion of hematopoietic precursor cell populations, ${ }^{39}$ suggesting that reduced expression of these genes in CBF $\beta-M Y H 11$ knockdown cells affects their self-renewal capacity.

\section{CONFLICT OF INTEREST}

The authors declare no conflict of interest.

\section{ACKNOWLEDGEMENTS}

We thank $E$ Megens, $K$ Berentsen and M Bergsma for assistance, C Logie for discussion and $\mathrm{H}$ Kerstens, $\mathrm{S}$ van Heeringen, $\mathrm{A}$ Brinkman and $\mathrm{K}$ Francoijs for bioinformatic support. U937-CBF $\beta$-MYH11 cells were a kind gift of T Pabst. This work was supported by the EU (ATLAS-221952; BLUEPRINT-282510), the Dutch Cancer Foundation (KWF KUN 2009-4527 and KUN 2011-4937) and the Netherlands Organization for Scientific Research (NWO-VIDI to JM).

\section{AUTHOR CONTRIBUTIONS}

The work presented here was carried out in collaboration between all authors. JHAM, AM, AS, PJ, AW, HR, FG, KP, SS, EV, MV and HGS designed methods and performed the experiments. JHAM, AM, AS, MV and HGS interpreted the results and wrote the manuscript and all authors contributed to the manuscript preparation. All authors have contributed to, seen, and approved the manuscript.

\section{DATABASE ACCESSION}

All ChIP-seq and RNA-seq data can be downloaded from the NCBI Gene Expression Omnibus (GEO) (http://www.ncbi.nlm.nih.gov/geo/) under accession number GSE46044.

\section{REFERENCES}

1 Appleford PJ, Woollard A. RUNX genes find a niche in stem cell biology. I Cell Biochem 2009; 108: 14-21.

2 Zeisig BB, Kulasekararaj AG, Mufti GJ, So CW. SnapShot: acute myeloid leukemia. Cancer Cell 2012; 22: 698-698, e691. 
3 Nimer SD, Moore MA. Effects of the leukemia-associated AML1-ETO protein on hematopoietic stem and progenitor cells. Oncogene 2004; 23: 4249-4254.

4 Shigesada K, van de Sluis B, Liu PP. Mechanism of leukemogenesis by the inv(16) chimeric gene CBFB/PEBP2B-MHY11. Oncogene 2004; 23: 4297-4307.

5 Liu P, Tarle SA, Hajra A, Claxton DF, Marlton P, Freedman M et al. Fusion between transcription factor CBF beta/PEBP2 beta and a myosin heavy chain in acute myeloid leukemia. Science 1993; 261: 1041-1044.

6 Springall $\mathrm{FH}$, Lukeis $\mathrm{RL}$, Tyrrell $\mathrm{V}$, Joshua $\mathrm{DE}$, lland $\mathrm{HJ}$. Identification of a nove CBFB-MYH11 fusion transcript in a patient with $\mathrm{AML}$ and inversion of chromo some 16. Leukemia 1998; 12: 2034-2035.

7 Schnittger S, Bacher U, Haferlach C, Kern W, Haferlach T. Rare CBFB-MYH11 fusion transcripts in $A M L$ with inv $(16) / t(16 ; 16)$ are associated with therapy-related $A M L$ M4eo, atypical cytomorphology, atypical immunophenotype, atypical additional chromosomal rearrangements and low white blood cell count: a study on 162 patients. Leukemia 2007; 21: 725-731.

8 Boeckx N, De Roover J, van der Velden VH, Maertens J, Uyttebroeck A Vandenberghe $\mathrm{P}$ et al. Quantification of CBFB-MYH11 fusion gene levels in paired peripheral blood and bone marrow samples by real-time PCR. Leukemia 2005; 19 1988-1990.

9 Castilla LH, Wijmenga C, Wang Q, Stacy T, Speck NA, Eckhaus M et al. Failure of embryonic hematopoiesis and lethal hemorrhages in mouse embryos heterozygous for a knocked-in leukemia gene CBFB-MYH11. Cell 1996; 87: 687-696.

10 Okuda T, van Deursen J, Hiebert SW, Grosveld G, Downing JR. AML1, the target of multiple chromosomal translocations in human leukemia, is essential for norma fetal liver hematopoiesis. Cell 1996; 84: 321-330.

11 Wang Q, Stacy T, Miller JD, Lewis AF, Gu TL, Huang X et al. The CBFbeta subunit is essential for CBFalpha2 (AML1) function in vivo. Cell 1996; 87: 697-708.

12 Castilla LH, Garrett L, Adya N, Orlic D, Dutra A, Anderson S et al. The fusion gene Cbfb-MYH11 blocks myeloid differentiation and predisposes mice to acute myelomonocytic leukaemia. Nat Genet 1999; 23: 144-146.

13 Castilla LH, Perrat P, Martinez NJ, Landrette SF, Keys R, Oikemus S et al. Identification of genes that synergize with Cbfb-MYH11 in the pathogenesis of acute myeloid leukemia. Proc Natl Acad Sci USA 2004; 101: 4924-4929.

14 Speck NA, Gilliland DG. Core-binding factors in haematopoiesis and leukaemia. Nat Rev Cancer 2002; 2: 502-513.

15 Van der Reijden BA, Massop M, Simons A, de Witte T, Breuning M, Jansen JH. The NDE1 gene is disrupted by the inv(16) in $90 \%$ of cases with CBFB-MYH11positive acute myeloid leukemia. Leukemia 2010; 24: 857-859.

16 Haferlach C, Dicker F, Kohlmann A, Schindela S, Weiss T, Kern W et al. AML with CBFB-MYH11 rearrangement demonstrate RAS pathway alterations in 92\% of all cases including a high frequency of NF1 deletions. Leukemia 2010; 24 1065-1069.

17 Zhao L, Melenhorst JJ, Alemu L, Kirby M, Anderson S, Kench M et al. KIT with D816 mutations cooperates with CBFB-MYH11 for leukemogenesis in mice. Blood 2011; 119: 1511-1521.

18 Liu PP, Hajra A, Wijmenga C, Collins FS. Molecular pathogenesis of the chromosome 16 inversion in the M4Eo subtype of acute myeloid leukemia. Blood 1995; 85: 2289-2302.

19 Lutterbach B, Hou Y, Durst KL, Hiebert SW. The inv(16) encodes an acute myeloid leukemia 1 transcriptional corepressor. Proc Natl Acad Sci USA 1999; 96 12822-12827.

20 Wee HJ, Voon DC, Bae SC, Ito Y. PEBP2-beta/CBF-beta-dependent phosphorylation of RUNX1 and p300 by HIPK2: implications for leukemogenesis. Blood 2008, 112: 3777-3787

21 Spruijt CG, Gnerlich F, Smits AH, Pfaffeneder T, Jansen PW, Bauer C et al. Dynamic readers for 5-(hydroxy)methylcytosine and its oxidized derivatives. Cell 2013; 152 1146-1159.

22 Yanagisawa K, Horiuchi T, Fujita S. Establishment and characterization of a new human leukemia cell line derived from M4E0. Blood 1991; 78: 451-457.

23 Andrews NC, Faller DV. A rapid micropreparation technique for extraction of DNA-binding proteins from limiting numbers of mammalian cells. Nucleic Acids Res 1991; 19: 2499.

24 van der Reijden BA, Massop M, Tonnissen E, van de Locht L, Muus P, de Witte T et al. Rapid identification of CBFB-MYH11-positive acute myeloid leukemia (AML) cases by one single MYH11 real-time RT-PCR. Blood 2003; 101: 5085-5086.

25 Martens $\mathrm{JH}$, Brinkman AB, Simmer F, Francoijs KJ, Nebbioso A, Ferrara $\mathrm{F}$ et al. PML-RARalpha/RXR alters the epigenetic landscape in acute promyelocytic leukemia. Cancer Cell 2010; 17: 173-185.
26 Martens JH, Mandoli A, Simmer F, Wierenga BJ, Saeed S, Singh AA et al. ERG and FLI1 binding sites demarcate targets for aberrant epigenetic regulation by AML1-ETO in acute myeloid leukemia. Blood 2012; 120: 4038-4048.

27 Yu M, Mazor T, Huang H, Huang HT, Kathrein KL, Woo AJ et al. Direct recruitment of polycomb repressive complex 1 to chromatin by core binding transcription factors. Mol Cell 2012; 45: 330-343.

28 Gardini A, Cesaroni M, Luzi L, Okumura AJ, Biggs JR, Minardi SP et al. AML1/ETO oncoprotein is directed to AML1 binding regions and co-localizes with AML1 and HEB on its targets. PLoS Genet 2008; 4: e1000275.

29 Zhang J, Kalkum M, Yamamura S, Chait BT, Roeder RG. E protein silencing by the leukemogenic AML1-ETO fusion protein. Science 2004; 305: 1286-1289.

30 Ernst J, Kellis M. ChromHMM: automating chromatin-state discovery and characterization. Nat Methods 2012; 9: 215-216.

31 Durst KL, Lutterbach B, Kummalue T, Friedman AD, Hiebert SW. The inv(16) fusion protein associates with corepressors via a smooth muscle myosin heavy-chain domain. Mol Cell Biol 2003; 23: 607-619.

32 Saeed S, Logie C, Francoijs KJ, Frige G, Romanenghi M, Nielsen FG et al. Chromatin accessibility, p300, and histone acetylation define PML-RARalpha and AML1-ETO binding sites in acute myeloid leukemia. Blood 2012; 120: 3058-3068.

33 Agrawal-Singh S, Isken F, Agelopoulos K, Klein HU, Thoennissen NH, Koehler G et al. Genome-wide analysis of histone $\mathrm{H} 3$ acetylation patterns in $\mathrm{AML}$ identifies PRDX2 as an epigenetically silenced tumor suppressor gene. Blood 2012; 119: 2346-2357

34 Whyte WA, Bilodeau S, Orlando DA, Hoke HA, Frampton GM, Foster CT et al. Enhancer decommissioning by LSD1 during embryonic stem cell differentiation. Nature 2012; 482: 221-225.

35 Wang Z, Zang C, Cui K, Schones DE, Barski A, Peng W et al. Genome-wide mapping of HATs and HDACs reveals distinct functions in active and inactive genes. Cell 2009; 138: 1019-1031.

36 Welboren WJ, van Driel MA, Janssen-Megens EM, van Heeringen SJ, Sweep FC, Span PN et al. ChIP-Seq of ERalpha and RNA polymerase II defines genes differentially responding to ligands. EMBO J 2009; 28: 1418-1428.

37 Verhaak RG, Wouters BJ, Erpelinck CA, Abbas S, Beverloo HB, Lugthart $S$ et al. Prediction of molecular subtypes in acute myeloid leukemia based on gene expression profiling. Haematologica 2009; 94: 131-134.

38 Helbling D, Mueller BU, Timchenko NA, Schardt J, Eyer M, Betts DR et al. CBFB-SMMHC is correlated with increased calreticulin expression and suppresses the granulocytic differentiation factor CEBPA in AML with inv(16). Blood 2005; 106: 1369-1375.

39 Chervinsky DS, Zhao XF, Lam DH, Ellsworth M, Gross KW, Aplan PD. Disordered T-cell development and T-cell malignancies in SCL LMO1 double-transgenic mice: parallels with E2 A-deficient mice. Mol Cell Biol 1999; 19: 5025-5035.

40 Varnum-Finney B, Purton LE, Yu M, Brashem-Stein C, Flowers D, Staats S et al. The Notch ligand, Jagged-1, influences the development of primitive hematopoietic precursor cells. Blood 1998; 91: 4084-4091.

41 Zhu J, Emerson SG. Hematopoietic cytokines, transcription factors and lineage commitment. Oncogene 2002; 21: 3295-3313.

42 Jankovic V, Ciarrocchi A, Boccuni P, DeBlasio T, Benezra R, Nimer SD. Id1 restrains myeloid commitment, maintaining the self-renewal capacity of hematopoietic stem cells. Proc Natl Acad Sci USA 2007; 104: 1260-1265.

43 Sands WA, Copland M, Wheadon H. Targeting self-renewal pathways in myeloid malignancies. Cell Commun Signal 2013; 11: 33.

44 Ptasinska A, Assi SA, Mannari D, James SR, Williamson D, Dunne J et al. Depletion of RUNX1/ETO in t(8;21) AML cells leads to genome-wide changes in chromatin structure and transcription factor binding. Leukemia 2012; 26: 1829-1841.

45 Adya N, Stacy T, Speck NA, Liu PP. The leukemic protein core binding factor beta (CBFbeta)-smooth-muscle myosin heavy chain sequesters CBFalpha2 into cytoskeletal filaments and aggregates. Mol Cell Biol 1998; 18: 7432-7443.

46 Kanno Y, Kanno T, Sakakura C, Bae SC, Ito Y. Cytoplasmic sequestration of the polyomavirus enhancer binding protein 2 (PEBP2)/core binding factor alpha (CBFalpha) subunit by the leukemia-related PEBP2/CBFbeta-SMMHC fusion protein inhibits PEBP2/CBF-mediated transactivation. Mol Cell Biol 1998; 18: 4252-4261.

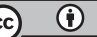

This work is licensed under a Creative Commons Attribution 3.0 Unported License. To view a copy of this license, visit http:// creativecommons.org/licenses/by/3.0/

Supplementary Information accompanies this paper on the Leukemia website (http://www.nature.com/leu) 